\title{
Ideación suicida, ansiedad, capital social y calidad de sueño en colombianos durante el primer mes de aislamiento físico por COVID-19
}

\section{Suicidal ideation, anxiety, social capital, and sleep quality in Colombians during the first month Physical Isolation by COVID-19}

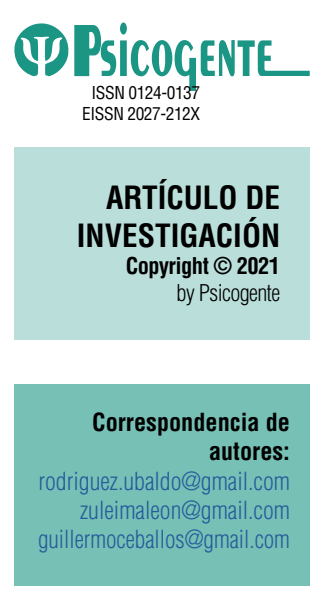

Recibido: 20-04-20 Aceptado: 03-12-20 Publicado: 01-01-21

Ubaldo Enrique Rodríguez de Avila (iD) - Zuleima Lucía León Valle (iD - Guillermo
Augusto Ceballos ospino (iD
Universidad del Magdalena

Resumen

Objetivo: explorar los niveles de ideación suicida, ansiedad, capital social y calidad de sueño en hombres y mujeres, según edades, en población colombiana durante el primer mes de Aislamiento Físico por pandemia de Covid-19.

Método: Estudio exploratorio y en línea, con 484 sujetos. Se utilizaron cuatro Escalas: Escala de Okasha para Suicidalidad (Okasha-IS), ZungSelf-Rating Anxiety Scale-15 (SAS-15), The Personal Social Capital Scale (PSCS) y Pittsburgh Sleep Quality Index (PSQI). Los instrumentos se registraron en la Web y se suministró mediante WhatsApp, Facebook y E-mail.

Resultados: Se encontró un índice de ideación suicida del 40\% entre leve a severa. 97\% de ansiedad leve a severa. Capital social personal regular (81\%) y $23 \%$ con poca calidad de sueño. La edad se asoció de manera inversa con valores de IS, SAS y PSQI $(p<0,01)$ y de manera directa con PSCS $(p<0,05)$. Sexo se correlacionó positivamente con SAS-15 $(p<0,01)$ y PQSI $(p<0,05)$. Mediante Análisis de Varianza se encontró diferencia significativa en hombres en los valores del IS y PQSI $(p<0,01)$ y en mujeres hay diferencia significativa en los valores de IS, SAS-15 y PQSI $(p<0,01)$ en diferentes grupos de edades.

Conclusiones: Las mujeres son más propensas a sentir ansiedad e ideación suicida asociados con Aislamiento Físico y bajo capital social, lo que puede desencadenar problemas psicológicos mayores. Los jóvenes menores de 20 años siguen siendo un grupo de mayor riesgo a padecer psicopatologías profundas, desencadenando suicidios. El presente estudio se adhiere a la idea de usar el término de distanciamiento Físico y no Aislamiento Social, dado que las relaciones sociales se mantienen desde niveles diferentes al contacto físico.

Palabras Clave: Ideación suicida, ansiedad, capital social, calidad de sueño, COVID-19 Y distanciamiento físico Colombia.

Abstract

Objective: to explore the levels of suicidal ideation, anxiety, social capital, and quality of sleep in men and women, according to age, in the Colombian population during the Covid-19 Pandemic Physical Isolation Method: Exploratory and online study with 484 subjects. Four Scales were used: Okasha Suicidality Scale (Okasha-IS), ZungSelf-Rating Anxiety Scale-15 (SAS-15), The Personal Social Capital Scale (PSCS) and Pittsburgh Sleep Quality Index (PSQI). The instruments were registered on the Web and provided through WhatsApp, Facebook and E-mail.

Results: A suicidal ideation rate of $40 \%$ was found between mild to severe. $97 \%$ of anxiety mild to severe. Regular personal social capital (81\%) and $23 \%$ with poor sleep quality. Age was inversely associated with 
IS, SAS and PSQI values ( $p<0,01)$ and directly with PSCS $(p<0,05)$. Sex was positively correlated with SAS-15 $(p<0,01)$ and PQSI $(p<0,05)$. By means of Analysis of Variance a significant difference was found in men in IS and PQSI values $(p<0,01)$ and in women there is a significant difference in IS, SAS-15 and PQSI values ( $p$ $<0,01$ ) in different age groups.

Conclusions: Women are more prone to feel anxiety and suicidal ideation associated with Physical Isolation and low social capital, which can trigger major psychological problems. Young people under 20 years of age continue to be a group at greater risk of suffering from profound psychopathologies, triggering suicides. In the present research it is recommended to use the term Physical Distancing and not Social Isolation, since social relationships are maintained from different levels than physical contact.

Key Words: Suicidal ideation, anxiety, social capital, sleep quality, COVID-19 and physical distancing Colombiam

\section{INTRODUCCIÓN}

A finales del último mes del año 2019 surgió un brote de neumonía de etiología desconocida en la ciudad de Wuhan (Hubei, China) y a partir de ahí un nuevo coronavirus fue identificado como el agente causal y posteriormente la enfermedad fue denominada COVID-19, considerado un pariente del síndrome respiratorio agudo severo (SARS) y del síndrome respiratorio de Medio Oriente (MERS), causado por un betacoronavirus Ilamado SARS-CoV-2 que afecta el tracto respiratorio inferior y se manifiesta como neumonía en humanos. (Sohrabia, Alsafib, O'Neilla, Khanb, Kerwanc, Al-Jabirc, Iosifidisa \& Aghad, 2020). Durante todo el mes de febrero de 2020, la pandemia de la COVID-19 en la provincia de Hubei, China, estaba en su apogeo, lo que requería el distanciamiento físico de la población. Esta circunstancia se extendió por todo el mundo y el Aislamiento Físico es la estrategia fundamental para contener la velocidad de expansión de la enfermedad y evitar que los sistemas de salud en países como Colombia colapsen.

Aunque investigaciones previas enfatizan en la importancia del contacto social para la propagación de enfermedades infecciosas en la crisis actual, (Bayer \& Kuhn, 2020; Mossong, Hens, Jit, Beutels, Auranen, Mikolajczyk, Massari, Salmaso, Scalia-Tomba, Liu, Eggo \& Kucharski, 2020; Bi, Wu, Mei, Ye, Zou, Zhang, Liu, Wei, Truelove, Zhang, Gao, Cheng, Tang, Wu, Wu, Sun, Huang, Sun, Zhang, Ma, Lessler \& Feng, 2020), las relaciones sociales son reconocidas como importantes determinantes sociales de la salud. (Berkman y Glass, 2014). Estudios demostraron que el aislamiento social se asocia con comportamientos de riesgo para la salud, como dieta poco saludable, fumar, alta ingesta de alcohol, inactividad física y múltiples comportamientos de riesgo para la salud. (Kaplan, Lazarus, Cohen \& Leu, 1991; Locher, Ritchie, Roth, Baker, Bodner, \&Allman, 2005; Shankar., McMunn, Banks \& Steptoe, 2011; Weyers, Dragano, Mobus, Beck, Stang, Mohlenkamp \& Siegrist, 2010). La mayoría de los estudios se centraron en el Aislamiento Social y la Soledad como factores independientes y algunos pocos se han centrado en la relación 
que existen entre ambos como factores asociados para la salud mental. (HolstAlgren, Ekholm, Nielsen, Kjær-Ersbøll, Kronborg-Bak \& Tanggaard-Andersen, 2020; Holt-Lunstad, Smith, Baker, Harris \& Stephenson, 2015; Shankar et al., 2011). Es así que el Aislamiento Social y la Soledad pueden ser considerados constructos diferentes, (Holst-Algren et al, 2020); algunas personas pueden sentirse solas a pesar de tener contacto social frecuente, mientras que otras con contacto infrecuente no se sienten solas. De ahí que la características sociales de interrelacionamiento se basan en la distancia física y no en el aislamiento social

De esta manera se verifica que la documentación científica demuestra que la soledad y el aislamiento social se asocian con un mayor riesgo de mortalidad, (Holst-Algren et al, 2020; Holt-Lunstad et al, 2015), incluido el comportamiento de salud (Berkman \& Glass, 2014; Berkman, Glass, Brissette \& Seeman, 2000; Cacioppo, Hawkley, Crawford, Ernst, Burleson, Kowalewski \& Berntson, 2002; Hawkley \& Cacioppo, 2010). De ahí que vivir solo y tener contactos sociales poco frecuentes con familiares y amigos son algunos de los marcadores del aislamiento social (Perissinotto \& Covinsky, 2014; Shankar, McMunn, Banks \& Steptoe, 2011). Mientras que el aislamiento social es una medida objetiva y cuantificable, (Holt-Lunstad et al., 2015), la soledad es la experiencia subjetiva de estar solo, relacionada con la insatisfacción con la discrepancia entre la frecuencia deseada y real de contacto social (De Jong Gierveld \& Havens, 2004; Peplau \& Perlman, 1982).

Por otro lado, la Ideación Suicida (IS) es un tema tocado desde múltiples perspectivas de la salud mental hasta características de los adolescentes. (Bahamón, Alarcón-Vásquez, Reyes-Ruiz, Uribe-Alvarado, García-Galindo \& Trejos-Herrera, 2018; Rodríguez, Rodrigues-deFrança Campos, ChionbacangaNafital, Ceballos-Ospino \& Paba-Barbosa, 2019). Las evidencias empíricas constatadas día a día sugieren que los problemas diversos pueden conllevar al suicidio, y para contrarrestarlo en momentos de Pandemia, se debe identificar en la fase de ideación, (pensamiento).

Diversos estudios han demostrado que grandes brotes de enfermedades infecciosas nuevas o graves están asociadas con niveles de ansiedad que pueden ser mucho mayores que el riesgo de infectarse o de mortalidad por infección (Leung, Lam, Ho, Ho, Chan, Wong \& Hedley, 2003). Además de los problemas de salud que trae consigo la Pandemia por la Covid-19, ya se tiene evidencia que trae igualmente sintomatología de ansiedad (Xiao, Zhang, Kong, Li \& Yang, 2020A, 2020B; Cai, Tu, Ma. Chen, Fu, Jiang \& Zhuang, 2020), ansiedad y depresión (Liu, Yang, Zhang, Xu, Dou, Zhang, 2020; Zhu,, Sun, 
Zhang, Wang, Fan, Yang, Xiao \& Li, 2020), además de un impacto importante a nivel psicológico o mental. (Liu, Ren, Yan, Li, Xu, Yu, Qu, Wang, Tian, Yang, Yao, Tan, Jiang \& Tan, 2020; Gao, Zheng, Jia, Chen, Mao, Chen, Wang, Fu \& Dai, 2020; Brooks, Webster, Smith, Woodland, Wessely, Greenberg \& Rubin, 2020; Guo, Liao, Wang, Li, Guo, Tong, Guan, Zhou, Wu, Zhang \& Gu, 2020; Liu, Liu \& Zhong, 2020).

De otra parte, hoy se sabe que el bienestar psicológico y la calidad de sueño se ven afectados por varios factores. Los factores sociales, como la carga económica, el apoyo familiar, el apoyo social y el capital social, también son factores importantes. (Mehnert, Lehmann, Graefen, Huland \& Koch, 2010). Varios estudios recientes han investigado la influencia de los factores sociales en la salud mental. (Guruge, Thomson, George \& Chaze, 2015). Pero hay poca investigación sobre la relación entre el capital social y la salud. (McDonald, 2018; Prati \& Pietrantoni, 2010).

El concepto de capital social fue propuesto por primera vez por el sociólogo francés, Portes, en 1980 (Portes, 1998) y en 1997, Lynch desarrolló el concepto de capital social como la voluntad de generar cohesión social, confianza y participación en actividades comunitarias. (Xiao, Zhang, Kong, Li \& Yang, 2020A, 2020B; Lynch \& Kaplan, 1997). Los estudios hacen distinción entre el apoyo social y el capital social. El apoyo social representa el tamaño y la fuente de las redes sociales de personas que ayudan a otros, así como las funciones de apoyo emocionales, materiales e informativas.(Khazaeian, Kariman, Ebadi \& Nasiri, 2017).

El capital social incluye confianza social, pertenencia y participación social. El efecto del capital social sobre el bienestar psicológico ha sido demostrado por estudios previos. (Harpham, Grant \& Rodríguez, 2017).

Al mismo tiempo, la calidad del sueño es un indicador clave de la salud, de ajuste emocional y de desempeño en general de los individuos. (MachadoDuque, Echeverri-Chabur \& Machado-Alba, 2015; Niño, Barragán, Ortiz, Ochoa \& González 2018; Prichard \& Hartmann, 2019). La buena calidad del sueño mantiene una función inmune óptima para prevenir infecciones. (Lange, Dimitrov \& Born, 2010). Es así que el bienestar psicológico como la ansiedad y el sueño, así como factores socioculturales se ven afectados igualmente por factores sociales significativos. (Yao, Yu, Cheng \& Chen, 2008; Brugha, 1990; Prati \& Pietrantoni, 2010; Kent de Grey, Uchino, Trettevik, Cronan, \& Hogan, 2018; Peng, Lee, Tsai, Yang, Morisky, Tsai, Weng \& Lyu, 2010). Estudios actuales han informado la relación de factores psicológicos 
como la ansiedad, donde se incluye la ideación suicida como síntoma psicológico importante, el estrés influyen en la calidad del sueño. (Bandura, 1977; Dahlgren, Kecklund \& Akerstedt, 2005; Chen, Zhang \& Xu, 2019).

Un estudio realizado en China, (Yuan, Liao, Huang, Jiang, Zhang, Wang \& Zhao, 2020), durante un período de dos semanas, demostró que trabajadores de salud de primera línea y otros habitantes de la provincia de Hubei se mostraron menos ansiosos por la epidemia de COVID-19, pero la calidad del sueño no mejoró. Los investigadores señalan que a pesar de la conciencia pública, existen niveles de ansiedad que afectan la calidad de vida durante las epidemias, incluidos los períodos de cuarentena de la población. Por lo tanto, la educación sanitaria debe combinarse con el asesoramiento psicológico para personas vulnerables.

La pandemia de COVID-19 se ha convertido en un factor estresante, particularmente porque se trata de una nueva infección viral que no tiene una vacuna y solo puede tratarse de acuerdo a los síntomas en la actualidad. En Colombia, no hemos encontrado estudios robustos sobre el papel del capital social en el bienestar, tampoco la manera cómo se manifiesta la ideación suicida y la ansiedad en los individuos sometidos a aislamiento físico, afectando incluso la calidad del sueño, especialmente en el contexto de enfermedades infecciosas agudas. Por lo tanto, este estudio tuvo como objetivo explorar los niveles de la ideación suicida, de la ansiedad, del capital social y la calidad de sueño en hombres y mujeres, segmentados por edades como factores de riesgo, en población colombiana durante el Aislamiento Físico por pandemia de Covid-19. De esta manera, la hipótesis exploratoria, es que existen diferencias significativas entre los niveles de ideación suicida, ansiedad y capital social entre hombres y mujeres, así como en diferentes rangos de edad.

\section{MÉTODO}

\subsection{Diseño}

Estudio de tipo cuantitativo, exploratorio y en línea. Los instrumentos se registraron en la Web y se suministró mediante WhatsApp, Facebook y Mail. El estudio se desarrolló entre las 14:00 horas del 29 de marzo de 2020 hasta las 14:00 horas del 06 de abril de 2020 (hora local colombiana), en el periodo de Aislamiento Físico obligatorio, decretado por el Gobierno Colombiano por la Pandemia por Covid-19. 


\subsection{Instrumentos}

A continuación, se presentan los diferentes instrumentos utilizados:

\subsubsection{Escala de Okasha (1981) para Suicidalidad (IS).}

Este instrumento mide el índice de Ideación Suicida y consta de cuatro ítems, los cuales se responden en una escala tipo Likert de 1 a 4 (Nunca, Casi nunca, Algunas veces y Muchas veces). En el presente estudio se promedia la puntuación total para obtener los rangos de IS ( $<1=$ Normal. 1,01-2= Leve a moderada. 2,01-3= Marcada a severa. 3,01-4= Extrema). En el Análisis Factorial Exploratorio (AFE) el Alfa de Cronbach ( $\alpha$ ) del IS y el coeficiente $\mathrm{KMO}$, o adecuación de la muestra de medición de Kaiser-Meyer-Olkin (Kaiser, 1974) fueron adecuados $(\alpha=0,85 ; \mathrm{KMO}=0,793)$. La prueba de esfericidad de Bartlett igualmente fue adecuada $\left[X^{2}(6)=583,504 ; p<0,01\right]$ y la varianza total explicada para un único factor identificado fue de $62,83 \%$. Lo que permite concluir un buen desempeño psicométrico de la Escala IS. Lo que confirma un buen ajuste psicométrico.

\subsubsection{ZungSelf-Rating Anxiety Scale-15 (SAS-15) versión español:}

La SAS es una medida de ansiedad diseñada por Zung (1971) para cuantificar el nivel de ansiedad para los sujetos que experimentan sus síntomas relacionados. Es una prueba autoadministrada que originalmente tiene 20 preguntas; cada pregunta se califica en una escala de 1-4 (Nunca, Algunas veces, Casi siempre y Siempre). Es una escala que está siendo usada en la comunidad científica desde sus inicios y actualmente también está siendo utilizada en estudios por la coyuntura de la Pandemia por la Covid-19. (Xiao et al, 2020A, 2020B). En el presente estudio se promedia la puntuación total para obtener los rangos de SAS-15 $(<1=$ Normal. 1,01-2= Leve a moderada. 2,01-3= Marcada a severa. 3,01-4= Extrema). Mediante un pilotaje previo en el participaron 449 sujetos, el AFE permitió reducir la Escala a 15 factores con un ajuste Psicométrico adecuado, $\alpha=0,81$ con $\mathrm{KMO}=0,876$ y la prueba de esfericidad de Bartlett igualmente fue adecuada $\left[X^{2}(119)=1179 ; p<0,01\right]$ y la varianza total explicada con un único factor identificado fue de $27,9 \%$. Lo que confirma un buen ajuste psicométrico.

\subsubsection{The Personal Social Capital Scale (PSCS) versión español}

La PSCS es una versión China (Chen, Stanton, Gong, Fang \& Li, 2009) basada en un marco conceptual de que el capital social es la parte de las conexiones de red de una persona que son confiables, recíprocas y ricas en recursos. 
(Bourdieu, 1986; Coleman, 1988; De Silva, McKenzie, Harpham \& Huttly, 2005; Harpham, Grant \& Thomas, 2002; Putnam, 1995). Creemos que no existe una versión en español de la PSCS; por lo menos en nuestra búsqueda no hemos encontrado. Esta versión consta de 42 ítems que medían 10 subconstructos, (factores):cinco subconstructos, que miden el capital social de enlace y otros cinco, que miden el capital social de puente. Las respuestas son dadas tipo Likert de manera inversa de 4 hasta 0 (Muchos, Más que el promedio, Menos que el Promedio, Promedio y Pocos, para dos subconstructos. Todas, La mayoría, Algunos, Pocos, Ninguno, para ocho subconstructos). Tanto para los subconstructos como el índice General del PSCS, se promediaron las puntaciones para obtener los rangos del PSCS en Español $(<1=$ Bajo. 1,01-2=Regular. 2,01-3=Moderado. 3,01-4=Óptimo). La evaluación psicométrica estricta para la escala original China, incluido el análisis factorial confirmativo, indicó que el PSCS tenía una fiabilidad excelente, una validez de estructura clara y una validez predictiva adecuada. (Chen et al., 2009). En la presente investigación, el AFE comprobó un $\alpha=0,94$ y un $K M O=0,89$ y la prueba de esfericidad de Bartlett igualmente fue adecuada $\left[X^{2}(861)=13524,551 ; p<0,01\right]$ y la varianza total explicada con diez factores identificados fue de $69,4 \%$. Lo que confirma un buen ajuste psicométrico.

\subsubsection{Pittsburgh Sleep Quality Index (PSQI) versión español}

EI PSQI (Buysse et al., 1989) contiene un total de 19 cuestiones, agrupadas en 10 preguntas. Las 19 cuestiones se combinan para formar siete áreas, (PSQI1: Calidad subjetiva del sueño, PSQI2: Latencia del sueño, PSQI3: Duración del sueño, PSQI4: Eficiencia habitual del sueño, PSQI5: Perturbación del sueño, PSQI: Medicación para dormir, PSQI7: Disfunción durante el día), con su puntuación correspondiente, cada una de las cuales muestra un rango comprendido entre 0 y 3 puntos. En todos los caso una puntuación de " 0 " indica facilidad, mientras que una de " 3 " indica dificultad severa, dentro de su respectiva área. La puntuación de las siete áreas se suman finalmente para dar una puntuación global del PSQI (PSQI-Total), que oscila entre 0 y 21 puntos. " 0 " indica facilidad para dormir y " 21 " dificultad severa en todas las áreas. Estos valores, se agruparon didácticamente en cuatro rangos: 1=Facilidad severa (1-5 puntos), Facilidad moderada (6-10 puntos), Dificultad moderada (11-15), Dificultad severa (16-21 puntos). En la presente investigación, el AFE comprobó un $\alpha=0,724$ y un $K M O=0,784$ y la prueba de esfericidad de Bartlett igualmente fue adecuada $\left[\mathrm{X}^{2}(21)=674,183 ; \mathrm{p}<0,01\right]$ y la varianza total explicada de $39,6 \%$. Lo que confirma un buen ajuste psicométrico. 


\subsection{Participantes}

Participaron inicialmente 550 sujetos. Seleccionados por medio de muestro por conveniencia. Se excluyeron 66 sujetos por ser habitantes de países diferentes a Colombia. La muestra quedó conformada por 484 sujetos colombianos, con edades entre 16 y 70 años $(M=30 ; D E=11)$. Se reunieron en cuatro grupos de edades, en relación a los diferentes segmentos de control ante el Aislamiento Físico por la Pandemia por Covid-19 en Colombia: Grupo $1(24,4 \%)$ : $<20$ años de edad. Grupo 2 (37,6\%): 21-30 años de edad. Grupo 3 (20\%): 31-40 años de edad. Grupo 4 (18\%): >40 años de edad. El 26\% fueron hombres y el $74 \%$ mujeres. El $70 \%$ aproximadamente moran en el Caribe Colombiano y el $30 \%$ en el resto del país. La desproporción en el número de hombres y mujeres, se debe al tipo de muestreo y no a otra situación particular

\subsection{Análisis de datos}

Análisis descriptivo porcentual. Para todas las variables interválicas o proporcionales se estimó la media (M) y desviación estándar (DE). A estas variables se les aplicó la prueba de Kolmogorov-Smirnov para conocer la distribución de las mismas. En todas las variables se comprobó la distribución no normal. Por esto, se decidió realizar la estimación de correlaciones de Spearman. Para la variable dicotómica (sexo) se calculó la correlación Tau-b de Kendall. Las variables Independientes para este estudio son Sexo y Edad, y las variables Ansiedad, Ideación Suicida, Capital Social y Calidad de Sueño son tratadas como variables dependientes. Para todas las variables se verificó la varianza inter-grupos, mediante el test de Kruskal-Wallis y U Mann-Whitney con probabilidad ajustada como análisis post hoc. Todo el análisis estadístico se realizó en el programa IBM-SPSS versión 22.

\subsection{Consideraciones éticas}

La investigación se hizo en consonancia con la Resolución 8430 que reglamentó la investigación en salud y con las normas precisas del Colegio Colombiano de Psicólogos, Conforme a la ley 1090 de 2006. Se contó con la firma de consentimiento informado en línea y el manejo confidencial de toda la información recogida.

\subsection{Procedimiento}

Se definió el problema, se creó el cuestionario en línea, de distribuyó mediante WhatsApp, Facebook y E-mail, y desde las 14:00 horas del 29 de marzo de 2020 hasta las 14:00 horas del 06 de abril de 2020 (hora local colombiana) se recolectó la información. 


\section{RESULTADOS}

A continuación, se describen los datos relacionados a las variables estudiadas (ver tabla 1)

Tabla 1.

Estadísticos descriptivos de las variables

\begin{tabular}{|c|c|c|c|c|c|c|c|}
\hline \multirow[b]{2}{*}{ ESCALA } & \multirow[b]{2}{*}{ TOTAL } & \multicolumn{2}{|c|}{ SEXO } & \multicolumn{4}{|c|}{ EDAD (AÑOS) } \\
\hline & & $\begin{array}{c}\text { HOMBRE } \\
(\mathrm{N}=126)\end{array}$ & $\begin{array}{c}\text { MUJER } \\
(\mathrm{N}=358)\end{array}$ & $\begin{array}{c}<20 \\
(N=118)\end{array}$ & $\begin{array}{c}21-30 \\
(N=182)\end{array}$ & $\begin{array}{c}31-40 \\
(N=97)\end{array}$ & $\begin{array}{c}>40 \\
(\mathrm{~N}=87)\end{array}$ \\
\hline Okasha-IS (M;DE) & 1,$40 ; 0,61$ & 1,$29 ; 0,54$ & 1,$40 ; 0,61$ & 1,$63 ; 0,75$ & 1,$43 ; 0,59$ & 1,$20 ; 0,44$ & 1,$09 ; 0,22$ \\
\hline Normal (\%) & 58,2 & 63,5 & 56,4 & 37,3 & 52,2 & 75,3 & 80,5 \\
\hline Leve a moderada (\%) & 28,9 & 27 & 29,6 & 39 & 33 & 17,5 & 19,5 \\
\hline Marcada a severa (\%) & 10,5 & 7,1 & 11,7 & 16,9 & 13,2 & 7,2 & 0 \\
\hline Extrema (\%) & 2,3 & 2,4 & 2,2 & 6,8 & 1,6 & 0 & 0 \\
\hline SAS-15 (M;DE) & 1,$61 ; 0,40$ & 1,$45 ; 0,32$ & 1,$61 ; 0,40$ & 1,$75 ; 0,41$ & 1,$60 ; 0,38$ & 1,$46 ; 0,45$ & 1,$38 ; 0,26$ \\
\hline Normal (\%) & 2,5 & 3,2 & 2,2 & 0 & 1,6 & 6,2 & 3,4 \\
\hline Leve a moderada (\%) & 84,9 & 91,3 & 82,7 & 77,1 & 84,6 & 87,6 & 93,1 \\
\hline Marcada a severa (\%) & 12,4 & 4,8 & 15,1 & 22,9 & 13,2 & 6,2 & 3,4 \\
\hline Extrema (\%) & 2 & 0,8 & 0 & 0 & 0,5 & 0 & \\
\hline PSCS (M;DE) & 1,$69 ; 0,64$ & 1,$72 ; 0,59$ & 1,$69 ; 0,64$ & 1,$57 ; 0,58$ & 1,$75 ; 0,62$ & 1,$70 ; 0,67$ & 1,$77 ; 0,64$ \\
\hline Bajo (\%) & 14,3 & 9,7 & 15,9 & 22,9 & 9,3 & 15,5 & 11,8 \\
\hline Regular (\%) & 56,2 & 68,5 & 52 & 54,2 & 56,6 & 56,7 & 57,6 \\
\hline Moderado (\%) & 25,9 & 16,9 & 29,1 & 22 & 30,2 & 21,6 & 27,1 \\
\hline Óptimo (\%) & 0,4 & 4,8 & 3,1 & 0,8 & 3,8 & 6,2 & 3,5 \\
\hline PSOI-Total (M;DE) & $7,7: 4,02$ & 6,$81 ; 3,72$ & 7,$71 ; 4,02$ & 8,$61 ; 3,8$ & 7,$77 ; 3,9$ & 6,$70 ; 3,8$ & 6,$21 ; 4,01$ \\
\hline Facilidad severa (\%) & 36,4 & 39,7 & 35,2 & 21,2 & 32,6 & 49,5 & 50,6 \\
\hline Facilidad moderada (\%) & 40,4 & 42,9 & 39,4 & 44,9 & 43,6 & 35,1 & 33,4 \\
\hline Dificultad moderada (\%) & 20,3 & 15,9 & 21,8 & 28,8 & 21,5 & 13,4 & 13,8 \\
\hline Dificultad severa (\%) & 2,9 & 1,6 & 3,4 & 5,1 & 2,2 & 2,1 & 2,3 \\
\hline
\end{tabular}

$M=$ Media. $D E=$ Desviación Estándar

Se verificaron nueve correlaciones significativas entre las variables estudiadas.

Al mismo tiempo se encontró diferencia significativa en relación al Sexo en las variables Ansiedad (SAS-15) y Calidad del Sueño (PSQI-Total) y en relación a Edad se encontró diferencia significativa para Índice de Suicidio (Okasha-IS), Ansiedad (SAS-15) y Calidad de Sueño (PSQI-Total). (Ver tabla 2). 
Tabla 2.

Correlación yvarianza para Sexo y Edad

\begin{tabular}{|c|c|c|c|c|c|c|c|c|}
\hline \multirow{3}{*}{ ESCALA } & $\begin{array}{c}\text { COEFI- } \\
\text { CIENTE DE }\end{array}$ & & & & \multicolumn{4}{|c|}{ ANÁLISIS DE LA VARIANZA INTRA-GRUPO } \\
\hline & \multicolumn{3}{|c|}{ SEXO } & \multicolumn{2}{|c|}{ EDAD } & \multirow[b]{2}{*}{$\mathbf{P}$} & \multirow[b]{2}{*}{ X2 (GL) } & \multirow[b]{2}{*}{$\mathbf{P}$} \\
\hline & OKASHA-IS & SAS-15 & PSCS & PSQI-TOTAL & X2 (GL) & & & \\
\hline Okasha-IS & & & & & $3,228(1)$ & 0,073 & $58,161(3)$ & $<0,01^{* *}$ \\
\hline SAS-15 & $0,355^{* *}$ & & & & $16,277(1)$ & $<0,01^{* *}$ & $56,863(3)$ & $<0,01^{* *}$ \\
\hline PSCS &,- 029 &,- 062 & & & $0,087(1)$ & 0,768 & $6,534(3)$ & 0,08 \\
\hline PSQI-Total &, $319^{* *}$ &, $583^{* *}$ &,- 049 & & $4,265(1)$ & $0,039 *$ & $26,963(3)$ & $<0,01^{* *}$ \\
\hline Sexo & ,075 &, $154^{* *}$ &,- 011 &, $080^{*}$ & & & & \\
\hline Edad &,$- 353^{* *}$ &,$- 333^{* *}$ &, $099^{*}$ &,$- 223^{* *}$ & & & & \\
\hline
\end{tabular}

$X^{2}=$ Chi cuadrado de Kruskal-Wallis. $(\mathrm{g})=$ Grados de libertad. $\mathrm{p}=$ Probabilidad

$$
*=p<0,05 . * *=p=0,01
$$

Agrupados los Sexos según las edades, solo se encontró diferencia significativa en la variable Ansiedad en los grupos de sujetos menores de 20 años de edad y los del rango de 21-30 años de edad. (Ver tabla 3).

Tabla 3.

Análisis de Varianza para Edad agrupada por Sexo

\begin{tabular}{|c|c|c|c|c|}
\hline \multirow[b]{2}{*}{ ESCALA } & \multicolumn{4}{|c|}{ EDAD (AGRUPADA PARA SEXO) } \\
\hline & $\begin{array}{c}<20 \\
(N=118)\end{array}$ & $\begin{array}{c}21-30 \\
(\mathrm{~N}=182)\end{array}$ & $\begin{array}{c}31-40 \\
(\mathrm{~N}=97)\end{array}$ & $\begin{array}{c}>40 \\
(\mathrm{~N}=87)\end{array}$ \\
\hline Okasha-IS [U (p)] & $1460(0,37)$ & $3713,5(0,08)$ & $934(0,52)$ & $743(0,85)$ \\
\hline SAS-15 [U (p)] & $1802(0,003)^{*}$ & $4129,5(0,003)^{*}$ & $1063(0,11)$ & $2817(0,66)$ \\
\hline $\operatorname{PSCS}[U(p)]$ & $5234(0,99)$ & $3582(0,24)$ & $691(0,12)$ & $629(0,35)$ \\
\hline PSQI-Total [U (p)] & $5321(0,59)$ & $3620(0,16)$ & $934(0,62)$ & $958(0,05)$ \\
\hline
\end{tabular}

U= Chi cuadrado de U Mann-Whitney. $\mathrm{p}=$ Probabilidad.

*= Probabilidad Significativa

Después de un análisis post hoc para muestras independientes con distribución no normal, con la probabilidad ajustada del Test de Kruskal-Wallis, segmentados por sexo y comparados los grupos por edades, se encontró diferencia significativa en hombres para las variables Ideación Suicida y Calidad de Sueño. Mientras en las mujeres, se encontró diferencia significativa en las variables Ideación Suicida, Ansiedad y Calidad de sueño en diferentes fajas etarias. (Ver figura 1). 
A

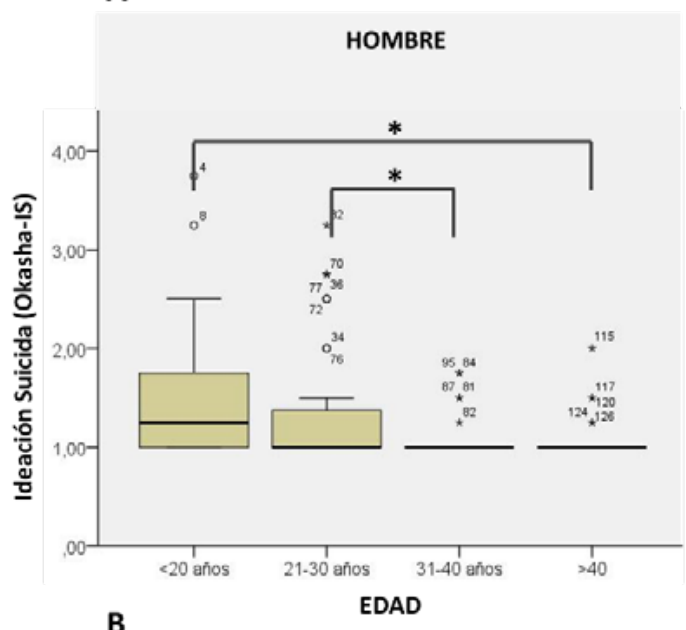

B

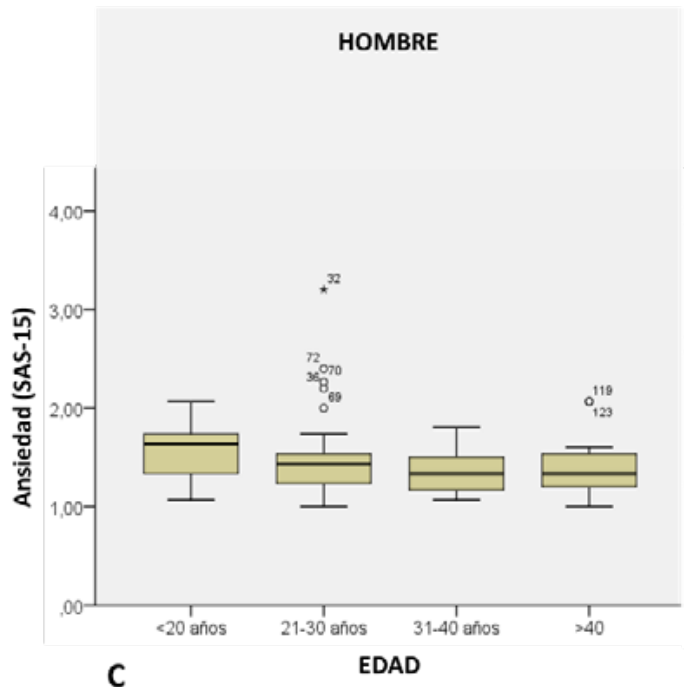

C

HOMBRE

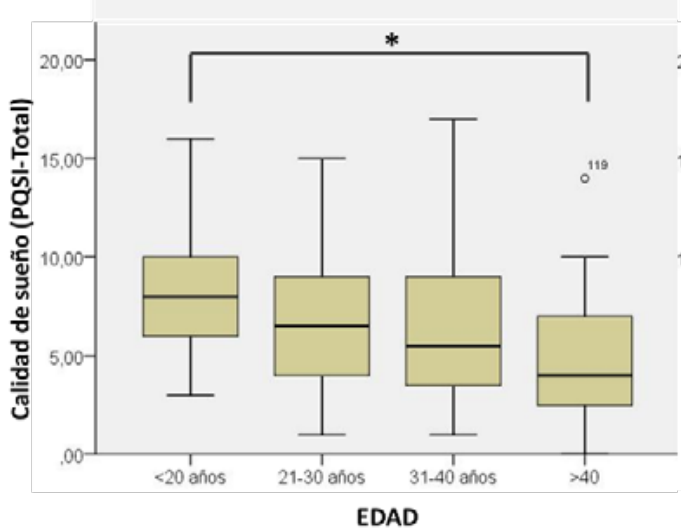

A1

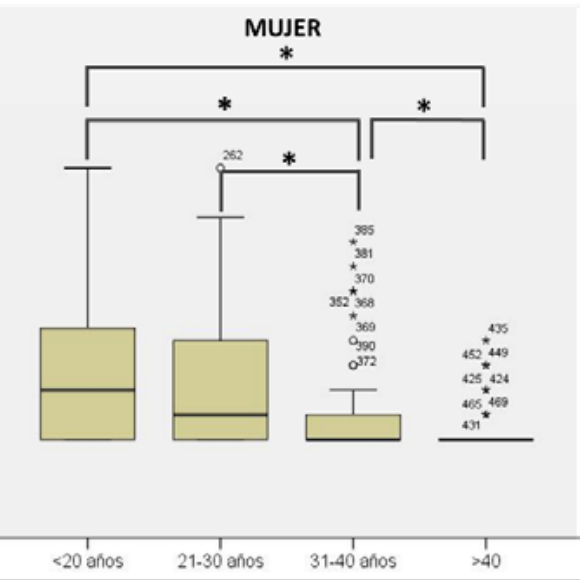

B1

EDAD

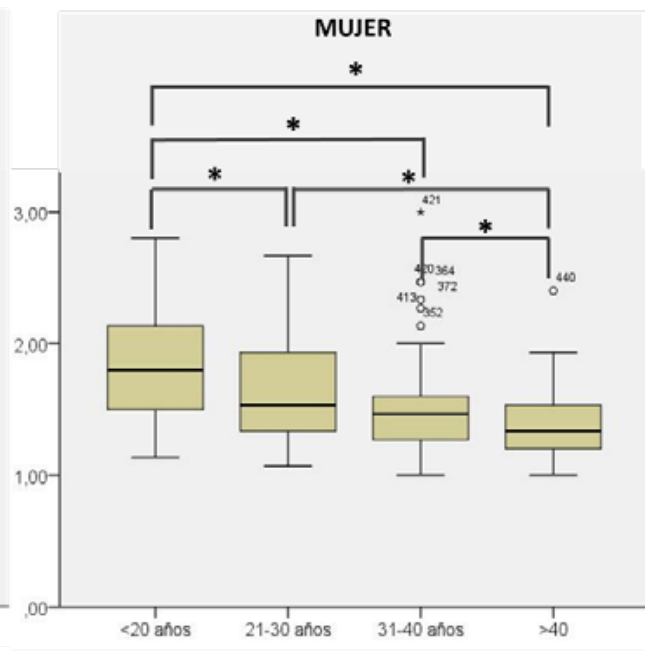

C1

MUJER

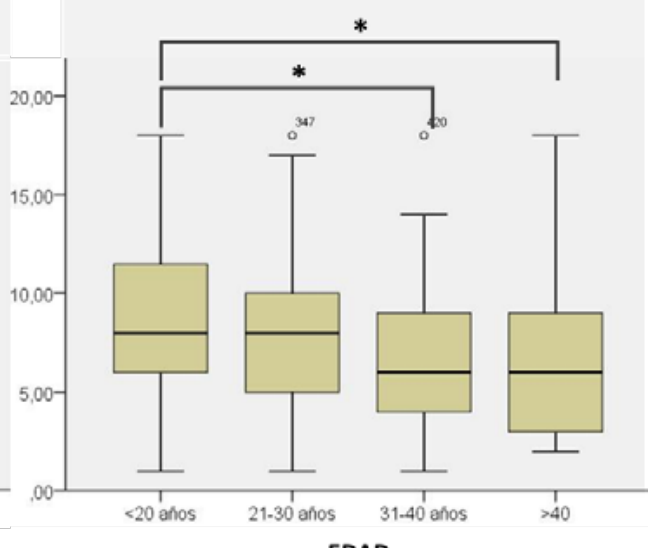

EDAD

Figura 1. Boxplot e post hoc para de las variables segmentadas por Sexo Vs Edad. 
A, B y C: Boxplot para hombres en las diferentes variables analizadas. A1, B1 y C1:Boxplot para mujeres en las diferentes variables analizadas. ${ }^{*}=p<0,01$ (se aplicó el test de Kruskal-Wallis y se determinó la probabilidad ( $p$ ) ajustada.

\section{DISCUSIONES}

Los resultados muestran que cerca del $40 \%$ de los sujetos presentan ideación suicida entre leve a severa y el $2 \%$ presentan ideación suicida severa. Las mujeres presentan el mayor índice de ideación suicida. $Y$ el subgrupo de menores de 20 años representa el mayor grupo de riesgo en ideación suicida extrema con un $6,8 \%$. Sin embargo, el análisis de la varianza no muestra diferencia significativa para los grupos de sexo, mientras que si se encontró diferencia significativa para los subgrupos de Edades. Mediante post hoc se confirma que la diferencia significativa entre las diferentes fajas etáreas, siendo los jóvenes menores de 20 años de edad, (hombre y mujeres), el mayor grupo de riesgo para la ideación suicida.

La puntuación del SAS muestra que cerca del $97 \%$ presenta ansiedad de leva a severa y igualmente las mujeres tienen los niveles más altos en relación a los hombres, lo que se confirma mediante el análisis de la varianza. El post hoc demuestra que existe diferencia significativa entre hombres y mujeres, sin embargo la probabilidad ajustada de Kruskal-Wallis demuestra que no existe diferencia significativa en los subgrupo de edades de hombres, mientras que para las mujeres, el mayor grupo de riesgo se encuentra en las mujeres más jóvenes, disminuyendo los niveles de ansiedad a medida que aumenta la edad. Estos resultados concuerdan con investigaciones recientes realizadas. (Xiao et al, 2020A, 2020B; Cai et al, 2020; Liu, D, et al, 2020; Zhu et al, 2020; Liu, X, et al 2020; Gao et al, 2020; Brooks et al, 2020; Guo et al, 2020; Liu, Liu \& Zhong, 2020).

Por otro lado, se encontró que el capital social personal se encuentra cerca del $81 \%$ en nivel regular a moderado, y las mujeres presentan mayores niveles. Las personas entre 31 y 40 años presentan los mejores índices en nivel óptimo en la puntuación del PSCS. Sin embargo, el análisis de la varianza no permite identificar diferencia significativa en los grupos de Sexo y Edad. No obstante, se puede considerar un posible factor predictivo de Ansiedad e Ideación Suicida y la calidad de sueño, tal como muestra resultados previos. (Bayer \& Kuhn, 2020; Liu et al, 2020; Bi et al, 2020).

El 23\% de los participantes presentan dificultad para dormir. El análisis de la varianza mediante la probabilidad ajustada de Kruskal-Wallis demuestra 
que existe diferencia significativa entre los subgrupos de edades menores de 20 años y mayores de 40 . Para las mujeres, el comportamiento de la puntuación en calidad de sueño es el mismo que los hombres, además de la diferencia entre menores de 20 años de edad y el grupo de 31-40 años de edad. Diferencia entre los sexos no se evidenció. Lo que comprueba que los jóvenes presentan mayor facilidad para dormir que los adultos. Los resultados concuerdan con informes previos. (Xiao et al, 2020A, 2020B).

\section{CONCLUSIÓN}

Se concluye que las mujeres colombianas estudiadas durante el primer mes de pandemia en general son más propensas a sentir ansiedad e ideación suicida, que combinados con el Aislamiento Físico y un bajo capital social, pueden desencadenar problemas mayores a nivel de ajuste psicológico.

Los jóvenes menores de 20 años, siguen siendo un grupo poblacional de mayor riesgo al padecer psicopatologías profundas, que pueden llegar a desencadenar suicidios. Es necesario diseñar programas de contención emocional y fortalecer los existentes, abriendo mecanismos de atención integral que incluyan el factor psicológico y social en la medida que se atiende la situación a nivel médico y epidemiológico.

Se recomienda para futuras investigaciones, ampliar otras variables de estudio que incluyan la formación familiar, sintomatología depresiva y otras de tipo sociológico, para determinar la relación entre las variables.

En el presente estudio se recomienda usar el término de Distanciamiento Físico y no Aislamiento Social, por cuanto esta última condición se presenta cuando una persona se aleja totalmente de su entorno de manera involuntaria. En el caso actual, las relaciones sociales se mantienen de manera on-line por diferentes "redes sociales". Es así que el Aislamiento Social es un constructo complejo que no concuerda con la acepción popularizada por sistemas divulgativos y gubernamentales, de conformidad a lo que expresan estudios previos. (Holst-Algren et al, 2020; Holt-Lunstad et al, 2015).

Conflicto de interés: Los investigadores declaran que no existe conflicto de interés alguno.

Agradecimientos: Al grupo de Cognición y Educación y al Grupo de Investigación Psicología de la salud y psiquiatría, ambos de la Facultad de Ciencias de la Salud, departamento de Psicología, Universidad del Magdalena, Santa Marta, Colombia.

Financiamiento: Universidad del Magdalena 


\section{REFERENCIAS}

Bahamón, M. J., Alarcón-Vásquez, Y., Reyes Ruiz, L., Uribe Alvarado, J. I., García Galindo, C., \&Trejos Herrera, A. M. (2018). Prácticas parentales como predictoras de la ideación suicida en adolescentes colombianos. Psicogente, 21(39), 50-61. https://doi.org/10.17081/psico.21.39.2821

Bandura, A. (1977). Self-efficacy: Toward a unifying theory of behavioral change. Psychol Rev, 84, 191-215. https://doi.org/10.1037//0033-295x.84.2.191

Bayer, C., \& Kuhn, M. (2020). Intergenerational ties and case fatality rates: A cross-country analysis. ECONtribute. https://selten.institute/wpcontent/ uploads/2020/03/ECONtribute_corona_bayer_kuhn_study_2020.pdf

Berkman, L. F., \& Glass, T. (2014). Social integration, social networks, social support, and health. Social epidemiology, 1, 137-173. http://dx.doi. org/10.4135/9781412952576.n192

Berkman, L. F., Glass, T., Brissette, I., \&Seeman, T. E. (2000). From social integrationto health: Durkheim in the new millennium. Social Science \& Medicine, 51(6), 843-857. https://doi.org/10.1016/S0277-9536(00)00065-4

Bi, O., Wu, Y, Mei, S., Ye, C., Zou, X., Zhang, Z., Liu, X., Wei, L., Truelove, S., Zhang, T., Gao, W., Cheng, C., Tang, X., Wu, X., Wu, Y., Sun, B., Huang, S., Sun, Y., Zhang, Y., Ma, T., Lessler, J., \& Feng, T. (2020). Epidemiology and transmission of covid-19 in Shenzhen China: Analysis of 391 cases and 1,286 of their close contacts. medRxiv, reprint https://doi.org/10.1101/2020.03.03.20028423.

Bourdieu, P. (1986). The forms of capital. In J. G. Richardson (Ed.), Handbook of theory and research for the sociology of education (pp. 241-258). Westport, CT: Greenwood Press. http://www.socialcapitalgateway.org/sites/socialcapitalgateway.org/files/data/paper/2016/10/18/rbasicsbourdieu1986-theformsofcapital.pdf

Brooks, S., Webster, R., Smith, L., Woodland, L., Wessely, S., Greenberg, N., \& Rubin, G. (2020). The Psychological Impact of Quarantine and How to Reduce It: Rapid Review of the Evidence. The Lancet, 395(10227), 912-920. https://doi. org/10.1016/S01406736(20)30460-8

Brugha, T. (1990). Social networks and social support. CurrOpinPsychiat, 3, 264-68. https://psycnet.apa.org/record/2011-28192-020

Buysse, D., Reynolds, C., Monk, T., Berman, S. \& Kupfer, D. (1989). The Pittsburgh sleep quality index: A new instrument for psychiatric practice and research. Psychiatry Research, 28(2), 193-213. https://doi.org/10.1016/0165-1781

Cai, H., Tu, B., Ma., Chen, L., Fu, L., Jiang, Y., \&Zhuang. (2020). Psychological Impact and Coping Strategies of Frontline Medical Staff in Hunan Between January and March 2020 During the Outbreak of Coronavirus Disease 2019 (COVID19) in Hubei, China. Med SciMonit, 26, e924171. https://doi.org/10.12659/MSM.924171

Coleman, J. S. (1988). Social capital in the creation of human capital. American Journal of Sociology, 94(S1), S95-S120. https://doi.org/10.1086/228943.

Chen, X., Stanton, B., Gong, J., Fang, X., \& Li, X. (2009). Personal Social Capital Scale: An instrument for health and behavioral research. Health Education Research, 24(2), 306-317. https://doi.org/10.1093/her/cyn020

Chen, X., Zhang, Y., \& Xu, X. (2019). The mediating effects of anxiety, self-efficacy and sleep quality on the relationship between doctor-patient's empathy and inflammatory marker in patients with ulcerative colitis. Med SciMonit, 25, 7889-97. https://doi.org/10.12659/MSM.917552

Cacioppo, J. T., Hawkley, L. C., Crawford, L. E., Ernst, J. M., Burleson, M. H., Kowalewski, R. B., ... Berntson, G. G. (2002). Loneliness and health: Potential mechanisms. 
Psychosomatic Medicine, 64(3), 407-417. https://www.ncbi.nlm.nih.gov/ pubmed/12021415

Dahlgren, A., Kecklund, G., \&Akerstedt, T. (2005). Different levels of work-related stress and the effects on sleep, fatigue and cortisol. Scand J Work Environ Health, 31, 277-285. https://www.jstor.org/stable/40967503?seq=1

De Jong Gierveld, J., \& Havens, B. (2004). Cross-nationalcomparisons of social isolation and loneliness: Introduction and overview. Canadian JournalonAging, 23(2), 109-113. https://doi.org/10.1353/cja.2004.0021

De Silva, M. J., McKenzie, K., Harpham, T., \&Huttly, S. R. (2005). Social capital and mental illness: A systematic review. Journal of Epidemiology and Community Health, 59(8), 619-627. https://doi.org/10.1136/jech.2004.029678.

Gao, J., Zheng, P., Jia, Y., Chen, H., Mao, Y., Chen, S., Wang, Y., Fu, H., \& Dai, J. (2020). Mental Health Problems and Social Media Exposure During COVID-19 Outbreak. The Lancet, Available at SSRN: http://dx.doi.org/10.2139/ssrn.3541120

Guo, J. Liao, L., Wang, B., Li, X., Guo, L., Tong, Z., Guan, Q., Zhou, M., Wu, Y., Zhang, J., \&Gu, Y. (2020). Psychological Effects of COVID-19 on Hospital Staff: A National Cross-Sectional Survey of China Mainland. The Lancet,Available at SSRN http:// dx.doi.org/10.2139/ssrn.3550050

Guruge, S., Thomson, M., George, U., \&Chaze, F. (2015). Social support, social conflict, and immigrant women's mental health in a Canadian context: a scoping review.J PsychiatrMent Health Nurs. Nov22(9):655-67. https://doi.org/10.1111/ jpm.12216.

Harpham, T., Grant, E., \& Thomas, E. (2002). Measuring social capital within health surveys: Key issues. Health Policy and Planning, 17(1), 106-111. https://doi. org/10.1093/heapol/17.1.106.

Harpham, T., Grant, E., \& Rodriguez, C. (2017). Mental health and social capital in Cali, Colombia. SocSci Med, 2004; 58(11): 2267-2277. https://doi.org/10.1016/j. socscimed.2003.08.013

Hawkley, L. C., \&Cacioppo, J. T. (2010). Loneliness matters: A theoretical and empirical review of consequences and mechanisms. Annals of Behavioral Medicine, 40(2), 218-227. https://doi.org/10.1007/s12160-010-9210-8

Holst-Algren., M, Ekholm, O., Nielsen, L., Kjær-Ersbøll, A., Kronborg-Bak, C., \&Tanggaard-Andersen, P. (2020). Social isolation, loneliness, socioeconomic status, and health-risk behaviour in deprived neighbourhoods in Denmark: A cross-sectional study. SSM - PopulationHealth, 10 (2020), 1-9. https://doi. org/10.1016/j.ssmph.2020.100546

Holt-Lunstad, J., Smith, T. B., Baker, M., Harris, T., \& Stephenson, D. (2015). Loneliness and social isolation as risk factors for mortality: A meta-analytic review. Perspectives on Psychological Science, 10(2), 227-237. https://doi. org/10.1177/1745691614568352

Kaiser, H. F. (1974). An index of factorial simplicity. Psychometrika, 34, 31-36. https:// doi.org/10.1007/BF02291575

Kaplan, G. A., Lazarus, N. B., Cohen, R. D., \& Leu, D. J. (1991). Psychosocialfactors in the natural history of physical activity. American Journal of Preventive Medicine, 7(1), 12-17. https://www.ncbi.nlm.nih.gov/pubmed/1867895

Kent de Grey, R., Uchino, B., Trettevik, R., Cronan, S., \& Hogan, J.N. (2018). Social support and sleep: A meta-analysis. Health Psychol, 37, 787-98. https://doi. org/10.1037/hea0000628

Khazaeian, S., Kariman, N., Ebadi, A., \&Nasiri, M. (2017). The impact of social capital and social support on the health of female-headed households: a systematic review. Electron Physician, 9(12), 6027-6034. https://doi.org/10.19082/6027 
Lange, T., Dimitrov, S., \& Born, J. (2010). Effects of sleep and circadian rhythm on the human immune system. Ann NY AcadSci, 1193: 48-59. https://www.jimmunol. org/content/158/9/4454.long

Leung, G., Lam, T., Ho., L., Ho, S.Y., Chan, B,H., Wong, I.O., \& Hedley, A.J. (2003). The impact of community psychological responses on outbreak control for severe acute respiratory syndrome in Hong Kong. J Epidemiol Community Health, 57(11), 857 - 63. https://doi.org/10.1136/jech.57.11.857

Liu, C., Yang, Y., Zhang, X., Xu, X., Dou, Q., \& Zhang, W. (2020). The Prevalence and Influencing Factors for Anxiety in Medical Workers Fighting COVID-19 in China: A Cross-Sectional Survey. The Lancet, Available at SSRN: http://dx.doi.org/10.2139/ ssrn.3548781

Liu, D., Ren, Y., Yan, F., Li, Y., Xu, X., Yu, X., Qu, W., Wang, Z. Tian, B. Yang, F., Yao, Y., Tan, Y., Jiang, R., \& Tan, S. (2020). Psychological impact and predisposing factors of the 2019 coronavirus disease pandemic (COVID-19) on the general public in China. The Lancet, Available at SSRN: http://dx.doi.org/10.2139/ssrn.3551415

Liu, X., Liu, J., \& Zhong, X. (2020). Psychological State of College Students During COVID-19 Epidemic. The Lancet, Available at SSRN: http://dx.doi.org/10.2139/ ssrn.3552814

Liu, Y., Eggo, R., \&Kucharski, A. (2020). Secondary attack rate and superspreading events for sars-cov-2. The Lancet. 395, https://doi.org/10.1016/S01406736(20)30462-1

Locher, J. L., Ritchie, C. S., Roth, D. L., Baker, P. S., Bodner, E. V., \& Allman, R. M. (2005). Social isolation, support, and capital and nutritionalrisk in anoldersample: Ethnic and genderdifferences. Social Science \& Medicine, 60(4), 747-761. https://doi. org/10.1016/j.socscimed.2004.06.023

Lynch, W., \& Kaplan, GA. (1997). Understanding how inequality in the distribution of income affects health. J Health Psychol. 2(3), 297-314. https://doi. org/10.1177/135910539700200303

Machado-Duque, M., Echeverri-Chabur, J., \& Machado-Alba, J. (2015). Somnolencia diurna excesiva, mala calidad del sueño y bajo rendimiento académico en estudiantes de Medicina. Revista Colombiana de Psiquiatría, 44(3), 137-142. https://doi.org/10.1016/j.rcp.2015.04.002

McDonald, K (2018). Social support and mental health in LGBTQ adolescents: A review of the literature. Issues Ment Health Nurs, 39(1), 16-29. https://doi.org/1 $0.1080 / 01612840.2017 .1398283$

Mehnert, A., lehmann, C., Graefen, M., Huland, H., \& Koch, U. (2010). Depression, anxiety, post-traumatic stress disorder and health-related quality of life and its association with social support in ambulatory prostate cancer patients. European Journal of Cancer Care. 19, 736-745. https://doi.org/10.1111/j.13652354.2009.01117.x

Mossong, J., Hens, N., Jit, M., Beutels, P., Auranen, K., Mikolajczyk, R., Massari, M., Salmaso, S., Scalia-Tomba, G., Wallinga, J., Heijne, J., Sadkowska-Todys, M., \& Rosinska, M. (2008). Social contacts and mixing patterns relevant to the spread of infectious diseases, PLoS Medicine 5(3). https://doi.org/10.1371/journal. pmed.0050074

Niño, J., Barragán, M., Ortiz, J., Ochoa, M., \& González., H. (2018). Factores asociados con somnolencia diurna excesiva en estudiantes de Medicina de una institución de educación superior de Bucaramanga. RevistaColombiana de Psiquiatría, (260), 1-10. https://doi.org/10.1016/j.rcp.2017.12.002 
Okasha, A., Lotaif, F., \&Sadek, A. (1981). Prevalence of suicidal feelings in a sample of nonconsulting medical students. Acta PsychiatrScand, (63), 409-415. Recuperado de https://www.ncbi.nlm.nih.gov/pubmed/7315487

Peng, E:, Lee., M., Tsai, S., Yang, C.C., Morisky, D.E., Tsai, L.T., Weng, Y.L., \& Lyu, S.Y. (2010). Population-based post-crisis psychological distress: An example from the SARS outbreak in Taiwan. J Formos Med Assoc, 109, 524-32. https://doi. org/10.1016/S0929-6646

Peplau, L. A., \& Perlman, D. (1982). Loneliness: A sourcebook of currenttheory, research, and therapy. New York: Wiley.

Perissinotto, C. M., \&Covinsky, K. E. (2014). Living alone, sociallyisolatedorlonelywhat are wemeasuring?. Journal of General Internal Medicine, 29(11), 1429-1431. https://doi.org/10.1007/s11606-014-2977-8

Portes, A. (1998). Social capital: Its origins and applications in modern sociology Annu Rev Soc, 24(1), 1-24. https://www.annualreviews.org/doi/abs/10.1146/ annurev.soc.24.1.1

Prati, G., \& Pietrantoni, L. (2010). The relation of perceived and received social support to mental health among first responders: A meta-analytic review. J Community Psychol, 2010; 38(3): 403-17. https://doi.org/10.1002/jcop.20371

Prichard, J., \& Hartmann, M. (2019). Follow-up to Hartmann \& Prichard: Should universities invest in promoting healthy sleep? A question of academic and economic significance. Sleep Health, 5(4), 320-325. https://doi.org/10.1016/j. sleh.2019.01.006

Putnam, R. D. (1995). Tuning in, tuning out: The strange disappearance of social capital in America. Political Science and Politics, 28(4), 664-683. https://doi. org/10.2307/420517.

Rodríguez, U., Rodrigues-deFrança Campos, F., Chionbacanga-Nafital , A., CeballosOspino, G., \&Paba-Barbosa , C. (2019). Las inteligencias, emociones y cronotipo, ¿explican el desempeño académico en universitarios?. Educación Y Humanismo, 22(38), 1-21 https://doi.org/10.17081/eduhum.22.38.3636

Shankar, A., McMunn, A., Banks, J., \&Steptoe, A. (2011). Loneliness, social isolation, and behavioral and biologicalhealthindicators in olderadults. HealthPsychology, 30 (4), 377-385. https://doi.org/10.1037/a0022826

Sohrabia, C., Alsafib, Z., O'Neilla, N., Khanb, M., Kerwanc, A., Al-Jabirc, A., Iosifidisa, C., \&Aghad, R. (2020). World Health Organization declares global emergency: A review of the 2019 novel coronavirus (COVID-19). International Journal of Surgery, 76 (2020), 71-76. https://doi.org/10.1016/j.ijsu.2020.02.034

Weyers, S., Dragano, N., Mobus, S., Beck, E. M., Stang, A., Mohlenkamp, S., ...Siegrist, J. (2010). Poor social relations and adverse healthbehaviour: Strongerassociations in lowsocioeconomicgroups?. International Journal of Public Health, 55(1), 17-23. https://doi.org/10.1007/s00038-009-0070-6

Xiao, H., Zhang, Y., Kong, D., Li, S., \& Yang, N. (2020A).Social Capital and Sleep Quality in Individuals Who Self-Isolated for 14 Days During the Coronavirus Disease 2019 (covid-19) Outbreak in January 2020 in China. Med SciMonit, 26, e923921. https://doi.org/10.12659/MSM.923921

Xiao, H., Zhang, Y., Kong, D., Li, S., \& Yang, N. (2020B).The Effects of Social Support on Sleep Quality of Medical Staff Treating Patients with Coronavirus Disease 2019 (COVID-19) in January and February 2020 in China. Med SciMonit, 26, e923549. https://doi.org/10.12659/MSM.923549

Yao, K., Yu, S., Cheng, S., \& Chen, I. (2008). Relationships between personal, depression and social network factors and sleep quality in community-dwelling older adults. J Nurs Res, 16, 131-139. https://doi.org/10.1097/01.JNR.0000387298.37419.ff 
Yuan, S., Liao, Z., Huang, H., Jiang, B., Zhang, X., Wang, Y., \& Zhao, M. (2020). Comparison of the Indicators of Psychological Stress in the Population of Hubei Province and Non-Endemic Provinces in China During Two Weeks During the Coronavirus Disease 2019 (COVID-19) Outbreak in February 2020. Med SciMonit, 26, e923767. https://doi.org/10.12659/MSM.923767

Zhu, J., Sun, L., Zhang, L., Wang, H., Fan, A., Yang, B., Xiao, S., \& Li, W. (2020). Prevalence and influence factors of anxiety and depression symptoms in front-line medical personnel fighting against COVID-19 in Gansu. The Lancet, Available at SSRN: http://dx.doi.org/10.2139/ssrn.3550054

Zung, W. (1971). A Rating Instrument For Anxiety Disorders. Psychosomatics, 12(6), 371-379. https://doi.org/10.1016/S0033-3182(71)71479-0

Esta obra está bajo: Creative commons attribution 4.0 international license. El beneficiario de la licencia tiene el derecho de copiar, distribuir, exhibir y representar la obra y hacer obras derivadas siempre y cuando reconozca y cite la obra de la forma especificada por el autor o el licenciante.

\section{(cc) BY}

